\title{
Aortic wall gadolinium enhancement in thoracic aortic aneurysm patients
}

\author{
Hadas Shiran ${ }^{2 *}$, Bob S Hu' ${ }^{1}$ Michael V McConnell ${ }^{2}$, David Liang ${ }^{2}$ \\ From 16th Annual SCMR Scientific Sessions \\ San Francisco, CA, USA. 31 January - 3 February 2013
}

\section{Background}

Thoracic aortic aneurysm (TAA) disease is an often asymptomatic, insidious process that includes structural aortic changes, dilation, and eventually causes death. Aneurysm prevalence exceeds $4 \%$ and is the 15 th leading cause of death in the US. TAA disease is associated with congenital and acquired defects in the aortic wall including elastin derangements, increased collagen, glycosaminolycan/proteoglycan accumulation, and loss of smooth muscle cells. We aim to investigate

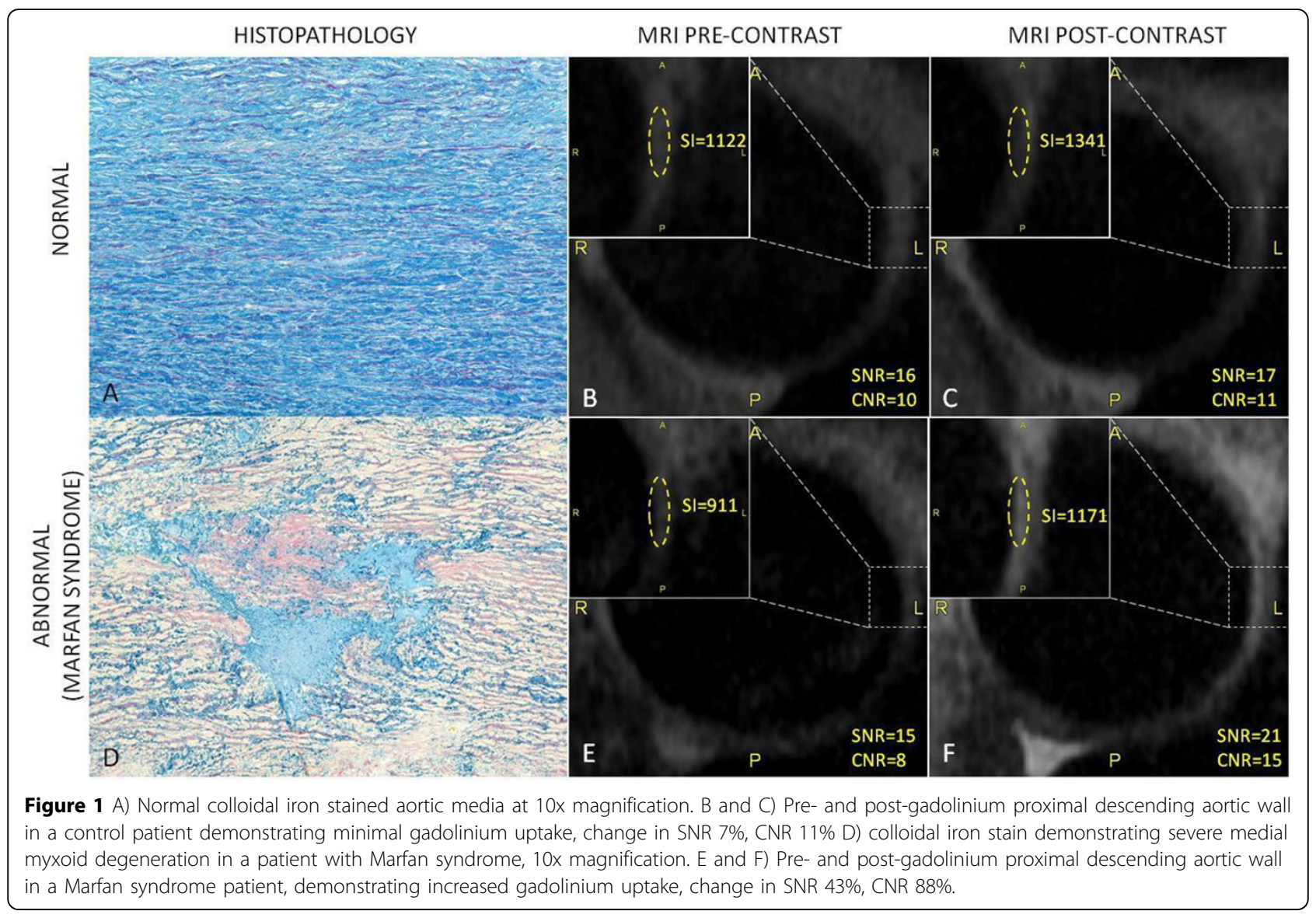

${ }^{2}$ Cardiovascular Medicine, Stanford University, Palo Alto, CA, USA
Full list of author information is available at the end of the article 


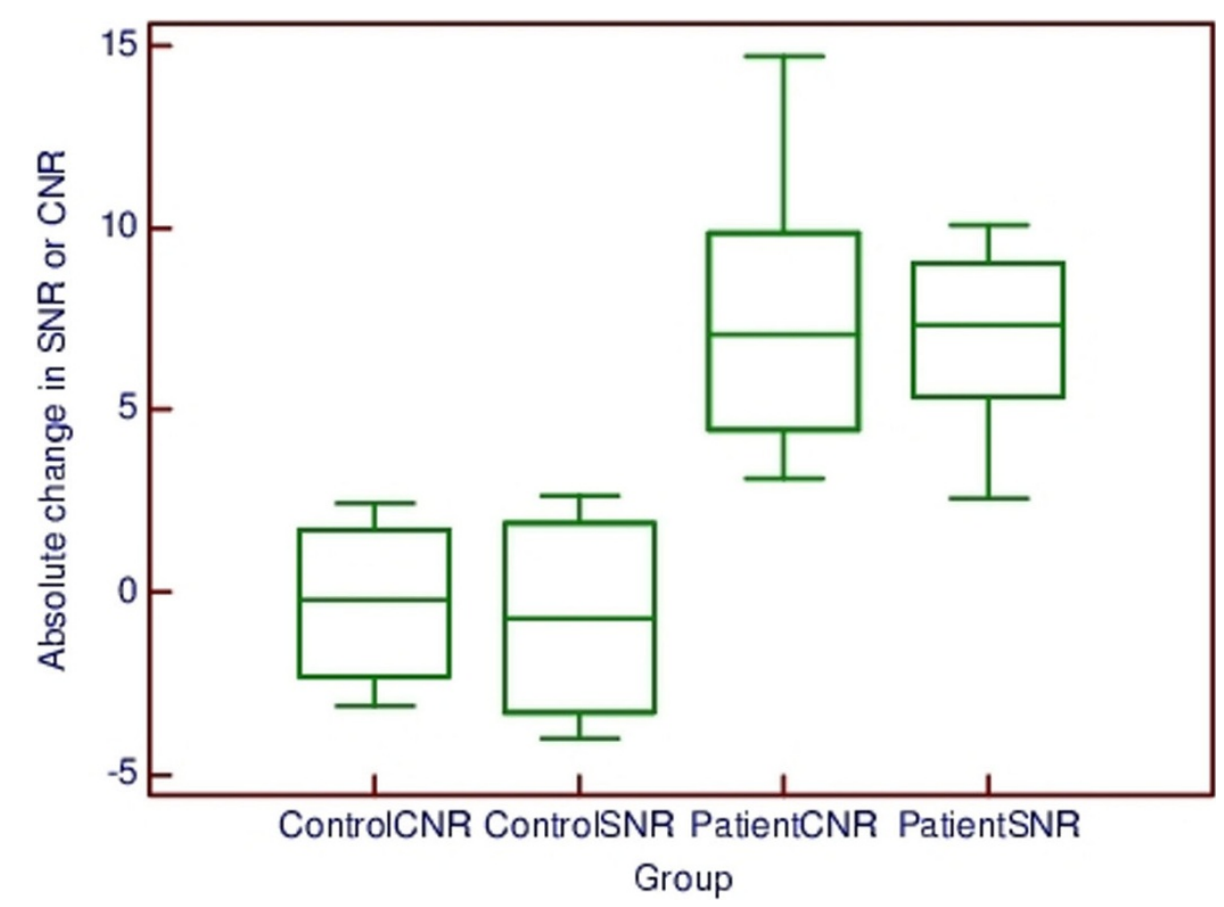

Figure 2 Comparison of SNR and CNR between patients and controls. Central box $=25-75$ percentile, middle line $=$ median. Whiskers $=$ range

these changes non-invasively by studying the uptake of gadolinium (Gd) in the diseased aortic wall. The distribution and accumulation of Gd depends on tissue blood flow, the size of the interstitial space, and permeability of capillaries, and has potential to help characterize severity of TAA disease.

\section{Methods}

We recruited patients undergoing cardiac MR or thoracic MRA for clinical care at Stanford University Hospital on a 1.5T GE scanner. We imaged a cross-section of the thoracic aorta before and after $\mathrm{Gd}$ administration, using T1-weighted double inversion recovery in 9 patients, 5 with connective tissue disease (e.g. Marfan syndrome) and 4 controls. We used a small field of view, $18 \mathrm{~cm}$, with matrix size $512 \times 224$, echo train length 16 , slice thickness $8 \mathrm{~mm}, 2 \mathrm{R}-\mathrm{R}$ intervals, ECG-gating and breath-holding were used, with a 30 second acquisition. The post-Gd images were acquired 5-7 minutes after injection. For each segment of aorta imaged, SNR and CNR were calculated for the aortic wall before and after contrast (CNR was calculated vs. lumen using a $7-10 \mathrm{~mm}^{2}$ region-of interest (ROI) in the wall and $50-55 \mathrm{~mm}^{2} \mathrm{ROI}$ in the lumen, as in Figure 1.

\section{Results}

We found significant post-Gadolinium enhancement of the aortic wall $(7.0+/-2.9$ absolute increase in SNR, 7.6 $+/$ - 4.4 increase in CNR) in patients with aneurysms and connective tissue disease (Figure 2). By comparison, control patients had minimal evidence of Gd uptake $(-0.7+/-3.1$ decrease in SNR, $-0.3+/-2.5$ decrease in CNR). $\mathrm{P}<0.05$ for SNR and CNR comparisons in patients versus controls.

\section{Conclusions}

Significant aortic wall Gd enhancement is seen in patients with connective tissue diseases and aneurysm formation and likely represents presence and severity of underlying tissue pathology.

\section{Funding}

None.

Author details

${ }^{1}$ Electrical Engineering, Stanford University, Palo Alto, CA, USA.

${ }^{2}$ Cardiovascular Medicine, Stanford University, Palo Alto, CA, USA.

Published: 30 January 2013

doi:10.1186/1532-429X-15-S1-E78

Cite this article as: Shiran et al:. Aortic wall gadolinium enhancement in thoracic aortic aneurysm patients. Journal of Cardiovascular Magnetic Resonance 2013 15(Suppl 1):E78. 